\title{
Analysing GPCR-ligand interactions with the fragment molecular orbital (FMO) method
}

Alexander Heifetz ${ }^{1 *}$, Tim James ${ }^{1}$, Michelle Southey ${ }^{1}$, Inaki Morao ${ }^{1}$, Dmitri G. Fedorov ${ }^{2}$, Mike

J. Bodkin ${ }^{1}$ and Andrea Townsend-Nicholson ${ }^{3}$

${ }^{1}$ Evotec (UK) Ltd., 114 Innovation Drive, Milton Park, Abingdon, Oxfordshire OX14 4RZ, United Kingdom

${ }^{2}$ Research Center for Computational Design of Advanced Functional Materials (CD-FMat), National Institute of Advanced Industrial Science and Technology (AIST), 1-1-1 Umezono, Tsukuba, Ibaraki 305-8568, Japan

${ }^{3}$ Institute of Structural \& Molecular Biology, Research Department of Structural \& Molecular Biology, Division of Biosciences, University College London, London, WC1E 6BT, United Kingdom

\section{Keywords:}

G-protein coupled receptors, GPCR, chemical interactions, pair-interaction energy, drugs, receptor, computational, modelling, QM, Quantum Mechanics, FMO, Fragment Molecular Orbitals method, CADD, Computer-Aided Drug Design, SBDD, Structure Based Drug Design, GAMESS, General Atomic and Molecular Electronic Structure System, PIEDA, Pair Interaction Energy Decomposition Analysis 


\section{Abstract}

G-protein coupled receptors (GPCRs) have enormous physiological and biomedical importance and therefore it is not surprising that they are the targets of many prescribed drugs. Further progress in GPCR drug discovery is highly dependent on the availability of protein structural information. However, the ability of X-ray crystallography to guide the drug discovery process for GPCR targets is limited by the availability of accurate tools to explore receptorligand interactions. Visual inspection and molecular mechanics approaches cannot explain the full complexity of molecular interactions. Quantum mechanics approaches (QM) are often too computationally expensive to be of practical use in time sensitive situations, but the fragment molecular orbital (FMO) method offers an excellent solution that combines accuracy, speed and the ability to reveal key interactions that would otherwise be hard to detect. Integration of GPCR crystallography or homology modelling with FMO reveals atomistic details of the individual contributions of each residue and water molecule towards ligand binding, including an analysis of their chemical nature. Such information is essential for an efficient structure-based drug design (SBDD) process. In this chapter, we describe how to use FMO in the characterisation of GPCR-ligand interactions. 


\section{Introduction}

1.1 G-protein coupled receptor - ligand interactions are fundamental to almost all processes occurring in living organisms. Not surprisingly, therefore, $34 \%$ of all drugs approved by the FDA (US Food and Drug Administration) act on this group of proteins [1]. What is surprising is that, collectively, these drugs only target around $13 \%$ of the 800 known GPCRs [2]. Thus, there is huge potential in terms of the number of new therapeutic targets within this family of receptors.

1.2 Further progress in drug discovery for GPCRs is highly dependent upon gaining an in depth understanding of the structure-activity relationships (SAR) and the individual interactions between the receptor and a small molecule (drug candidate) [2-5]. The efficiency and cost-effectiveness of the drug-design process can be increased by the availability of structural data regarding the target protein and by the reliability of the computational tools used for data exploration [3-5]. However, even with the crystal structure, "visual inspection" and the force field-based molecular mechanics (MM) calculations often used for the rationalization of ligand-protein potency cannot always explain the full complexity of the molecular interactions [6].

1.1. There is increasing evidence [6-9] that there are a number of non-intuitive interactions, such as $\mathrm{CH} / \pi[10,11]$, halogen/ $\pi$ [12], cation/ $\pi$ [13], non-classical $\mathrm{H}$-bonds [14] and others, which play important roles in protein-ligand binding but that are not sufficiently parameterized in most popular force fields (FF) [8]. Quantum mechanical methods have been employed to improve the reliability of the exploration of protein-ligand interactions $[15,16]$. Historically, the application of high level ab initio QM calculations was limited to molecular systems consisting of a small number of atoms, usually low molecular weight organic molecules. Recent and continuing advances in computer 
science have enabled the method to be applied to much larger biological molecules such as GPCRs [17].

1.2. The fragment molecular orbital method $[11,16,18]$ offers a considerable increase in computational speed over traditional QM methods [19]. One of the key advantages of the FMO approach is that the output from these calculations includes a list of the interactions formed between the ligand and the receptor along with a chemically intuitive breakdown of these interactions [17]. Such information is essential for medicinal chemists to be able to adopt a rational approach to the modification of lead compounds in order to enhance favourable interactions.

1.3. FMO works by partitioning a system into smaller fragments (Figure 1). For example, in a receptor, each residue can be represented as a fragment. Similarly, the ligand can be represented by a single or multiple fragments, as necessary. By performing QM calculations on the fragments, one is able to attain a high level of accuracy with increased efficiency.

$<$ FIGURE 1>

Figure 1. Workflow for FMO calculations and details on each of the PIE terms that are computed [17]. The electrostatic component arises from the Coulomb interaction between polarized charge distributions of fragments. The exchange repulsion term is derived from the interaction between fragments situated in close proximity and is always repulsive; it is due to the Pauli repulsion and is related to the overlap of two occupied orbitals. The charge transfer term arises from the interaction between occupied orbitals of a donor and unoccupied orbitals of an acceptor. The dispersion arises as the interaction between instantaneous dipole moments of two fragments, it is hydrophobic (non-polar) in nature and is obtained in PIEDA from the correlation energy of electrons. 
1.4. As illustrated in Figure 1, the pair interaction energy (PIE) between any two fragments calculated by FMO is the sum of four energy terms - electrostatic, exchange-repulsion, charge transfer and dispersion - and is calculated using pair interaction energy decomposition analysis (PIEDA) [20]. The electrostatic and charge transfer terms are predominant in salt-bridge, hydrogen bond and polar interactions, whilst the dispersion term generally corresponds to interactions that are predominantly hydrophobic in nature. The role of hydrophobic interactions is integral to biomolecular recognition but there is still no reliable non-QM predictive method for its quantification [6]. Exchange-repulsion is a high level QM term that quantifies the repulsion between electrons [17].

1.5. FMO is highly useful tool for structure-based drug design [11, 21, 22], as it provides an accurate and comprehensive list of strong, weak and repulsive interactions between the ligand and its surrounding residues. Such information is highly instructive in terms of modifications, scaffold replacement (scaffold hopping), linking (specifically in the case of fragment-based drug discovery) or extension of chemical moieties to form stronger or new interactions with the protein or alternatively to remove repulsions. FMO can also be applied in the analysis of ligand-water-protein networks to distinguish between energetically favourable and unfavourable water molecules, thus enabling the design of ligands that can interact with or displace certain waters.

1.6. Application of the FMO method in the hit-to-lead and lead optimization stages of drug discovery is a highly valuable approach for the design, evaluation and filtering of targets for synthesis as it can significantly decrease the effort and cost of chemical synthesis (for more details see chapter 3 of this book). 
1.7. As previously demonstrated [23], significant correlation between receptor-ligand affinities and FMO energy terms [24] indicates that the latter can be efficiently used as descriptors in QSAR modelling to predict the binding affinities of new molecules. FMO has been successfully applied in the discovery of novel Hsp90 inhibitors [21] and in many of our confidential drug discovery programs.

1.8. Reported examples [24] comparing FMO and MM have shown that the FMO method clearly outperformed force field based scoring functions and demonstrated a high correlation with experimentally measured values of protein-ligand affinity [23]. The key differentiator between the FMO and MM methods is that the former accounts for polarization and charge transfer effects $[11,25]$. The description of electrostatics in most force fields is based on static charges that neglect polarization and in systems such as proteins this is an approximation to the actual state. In addition, van-derWaals forces, despite being generally well parameterized on average, are not capable of detecting the directional nature of the dispersion terms involving halogens [26].

1.9. In this chapter, we describe a number of examples of the application of FMO to the exploration of receptor-ligand interactions. 


\section{Methods}

2.1. Several QM packages, including GAMESS [27], ABINIT-MP [28] and PAICS [29], contain modules for performing FMO calculations. A typical FMO-MP2 calculation on a ligand-receptor complex takes approximately $4 \mathrm{~h}$ on a $36 \mathrm{CPU}$ cluster to complete, significantly faster than the weeks to a month (or more) for traditional QM approaches that have been used for estimating binding energies. Recent developments in methodology and integration with the density-functional tight-binding (DFTB) approach [30] have further increased the throughput.

2.2. In FMO calculations, a biological system is partitioned into fragments (Figure 1) [11, 16]. Each residue is typically characterised as a fragment and the interaction energies reported herein correspond to actual amino acid residues as opposed to residue fragments. The ligand can be represented as a whole or subdivided; some ligands are very large and dividing such molecules into multiple fragments has the benefit of both reducing the computational cost and providing a more detailed analysis. An in depth description of the FMO strategy and methodology can be found in previously published reports $[11,16,20]$, including detailed mathematical formulae that are beyond the scope of this manuscript. 
2.3. An FMO calculation consists of the following steps: (a) Fragmentation (i.e., assigning atoms in a system to a fragment); (b) Fragment self-consistent field (SCF) calculations in the embedding polarizable potential, so that fragments mutually polarize each other in a self-consistent fashion whereby intra-fragment charge transfer and other quantum effects are accounted for; (c) Fragment pair SCF calculations, bringing in interfragment charge transfer; (d) Total property (energy, gradient, etc.) evaluation. By performing QM computations on fragments one can achieve high efficiency, often resulting in linear scaling. The FMO method has been parallelized for PC clusters [19]. By default we use the MP2 method (second order Møller-Plesset perturbation theory [31]) with the $6-31 G^{*}$ basis set. This basis set is often considered a good compromise between speed and accuracy [24].

2.4. As illustrated in Figure 1 , the pair interaction energy $\left(\Delta E_{i j}^{i n t}\right.$, or PIE) between fragments $i$ and $j$ is a sum of 4 terms: electrostatics $\left(\Delta E_{i j}^{e s}\right)$, exchange-repulsion $\left(\Delta E_{i j}^{e x}\right)$, charge transfer $\left(\Delta E_{i j}^{c t}\right)$ and dispersion $\left(\Delta E_{i j}^{d i}\right)$ :

$$
\Delta E_{i j}^{\mathrm{int}}=\Delta E_{i j}^{\mathrm{es}}+\Delta E_{i j}^{\mathrm{ex}}+\Delta E_{i j}^{c t}+\Delta E_{i j}^{\mathrm{di}}
$$

2.5. The PIE is not a difference between the energies of the receptor-ligand complex and the sum of the 'free' protein and ligand, but rather represents the 'strength' of the interaction between the ligand and protein residues in the complex. The $\Delta$ denotes the differences in total QM energy of a fragment pair $i j$ and two individual fragments $i$ and $j$, both computed in the receptor-ligand complex. In the present work, fragment $i$ is the ligand and the other $n$ fragments are receptor residues and water molecules. In the equations below there is no self-interaction (the sums exclude $j=i$ ). The total PIE calculated by the FMO method describes the stability of the receptor-ligand complex. This stability correlates with, but is not the same as, the binding energy [24]. The difference lies in the polarization factors - the ligand is polarized by the protein and 
vice versa [19]. Based on previous reports [11], we recommend that only interactions with an absolute PIE greater than or equal to $3.0 \mathrm{kcal} / \mathrm{mol}$ should be considered as significant. 


\title{
3. Notes
}

3.1 We originally applied the FMO method to investigate the interactions in 18 class A GPCRligand crystal structures, which represent different branches of the GPCR family and include both active and inactive states [23].

3.2 The purpose of this investigation was to explore the chemical nature of GPCR-ligand interactions and to test whether FMO can provide insights beyond the conventional 'visual inspection' or MM-based assessments [16]. We were also interested in exploring which of the three potentially attractive PIE terms (electrostatics, charge-transfer and dispersion) is dominant in the receptor-ligand binding and if there is a consensus among the whole class A GPCR family.

3.3 To illustrate the typical outcome of an FMO calculation we give five examples (Figures 26) taken from an earlier report [23]. The results are shown as panels where the left hand figures $(A)$ show 3D images of the receptor-ligand complexes. The carbon atoms of the ligands are shown in light orange and those of the receptors are coloured according to the PIE values calculated by FMO. Nitrogen atoms are shown in blue, oxygen in red, sulfur in yellow and chlorine in light green. The right hand plots (B) show the sorted PIE values of the most strongly interacting residues on the top and the PIEDA of these key interactions on the bottom. PIE terms: electrostatics, dispersion, charge-transfer and exchange repulsion are colour coded yellow, blue, red and green, respectively.

\author{
$<$ FIGURE 2>
}

Figure 2. Human Angiotensin II type 1 receptor $\left(A T_{1} R\right)$ in complex with ZD7155 (PDB entry 4YAY [32]) - $A T_{1} R$ is primarily involved in blood pressure regulation. Several antihypertensive drugs have been developed as $A T_{1} R$ blockers. However, the structural basis for $A T_{1} R$ ligand binding and function had previously been poorly understood. The recently developed method of serial femtosecond crystallography at an X-ray free-electron laser was successfully applied to determine the crystal structure of the human AT ${ }_{1} R$ - ZD7155 complex. FMO calculations detected 3 major interactions involved in ZD7155 binding, formed by the residues Tyr35 ${ }^{1.39}$, Trp84 2.60 and Arg1674.64. [32] The PIEDA analysis showed that the interactions formed by Tyr35 $5^{1.39}$ and Arg $167^{4.64}$ are mainly electrostatic albeit with a significant portion of dispersion. This balance of terms is characteristic of salt-bridges or hydrogen 
bonding [11]. On the other hand, the interaction with $\operatorname{Trp} 84^{2.60}$ is mainly driven by dispersion, which fits more with $\pi$-stacking or hydrophobic forces [11]. These results are consistent with published SDM data [32], which showed a salt-bridge with Arg1674.64, hydrogen bond with Tyr35 $5^{1.39}$ and face-to-face $\pi$-stack with Trp84 2.60 are critical for ZD7155 binding. The other six interactions formed by residues Ser105 ${ }^{3.29}$, Val108 ${ }^{3.32}$, Ser109 ${ }^{3.33}$, Phe182 ${ }^{\mathrm{ECL} 2}$, Ile288 ${ }^{7.39}$ and Tyr292 $2^{7.43}$ are also consistent with the published analysis of this crystal structure.

\section{<FIGURE 3>}

Figure 3. Human chemokine $\mathrm{CCR}_{5}$ receptor in complex with Maraviroc (PDB entry 4MBS [33]) - FMO calculations detected 11 significant interactions with ten residues - Tyr37 $37^{1.39}$, Trp86 ${ }^{2.60}$, Tyr108 ${ }^{3.32}$, Phe109 ${ }^{3.33}$, Phe112 ${ }^{3.36}$, Phe182 ${ }^{\mathrm{ECL} 2}$, Thr195 ${ }^{5.39}, \operatorname{Trp}^{248^{6.48}}$, Tyr251 ${ }^{6.51}$ and Glu2837.39 - and one water molecule; $\mathrm{HOH} 1207$. These interactions were consistent with published analysis of the crystal structure [33,34]. It is interested to note that, in contrast to other class A GPCRs, in this receptor the salt bridge with the ligand is formed by the negatively charged residue located at position 7.39 and not at 3.32. While all of these interactions were suggested in the analysis of the crystal structure, additional interactions with residues GIn194 $4^{5.38}$, Ile198 ${ }^{5.42}$, Thr2596.59 and Met287 ${ }^{7.43}$, postulated from published reports, were also characterised as significant by FMO.

\section{<FIGURE 4>}

Figure 4. Human serotonin $5-\mathrm{HT}_{1 \mathrm{~B}}$ receptor in complex with Ergotamine (PDB entry 4IAR [35]) - FMO calculations highlighted 13 significant interactions with residues Asp1293.32,

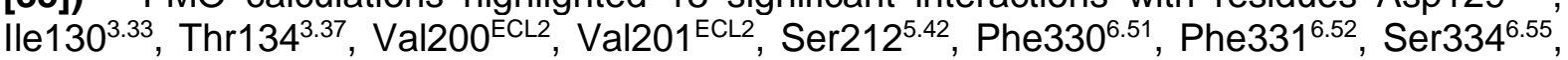
Met3376.58, Leu3487.32, Phe3517.35, Asp352 ${ }^{7.36}$ and Tyr3597.43. The vast majority of these interactions were consistent with published analysis of the crystal structure [36]. However, three non-classical hydrogen bonds [14] formed by the ligand with Ser212 ${ }^{5.42}$, Phe330 ${ }^{6.51}$ and Leu348 ${ }^{7.32}$ (backbone carbonyl) and two hydroxy- $\pi$ interactions [37] with residues Ser334.55 and Phe351 7.35 were newly detected by FMO. FMO also emphasized the two interactions of Asp352 $2^{7.36}$, which forms both strong electrostatic attraction with Ergotamine and, via its backbone atoms, a non-classical hydrogen bond with the ligand (marked as a dashed yellow line). An additional interesting interaction detected by FMO is the classical hydrogen bond formed between the backbone of $\mathrm{Val} 201^{\mathrm{ECL} 2}$ and the ligand (also marked as a dashed yellow line).

\section{$<$ FIGURE 5>}

Figure 5. Rat muscarinic $M_{3}$ muscarinic acetylcholine receptor in complex with Tiotropium (PDB entry 4DAJ [38]) - FMO calculations detected 10 significant interactions

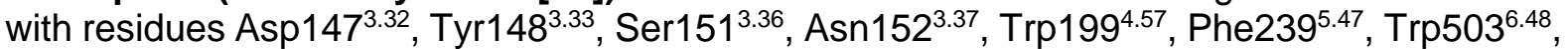
Tyr506 6.51, Asn5076.52 and Tyr5297.39. The majority of these interactions were suggested previously. [34, 36, 39] However, as in the $\mathrm{M}_{2}$ :QNB complex, the interactions with Asn1523.37 and Phe2395.47 were newly detected by FMO. The backbone nitrogen of Asn $152^{3.37}$ and a hydrogen from the methylene linker of Phe2395.47 form hydrophobic and non-classical hydrogen bonds [14], respectively, with the ligand (marked as dashed yellow lines). 
$<$ FIGURE 6>

Figure 6. Human histamine $\mathrm{H}_{1}$ receptor in complex with Doxepin (PDB entry 3RZE [40]) - FMO calculations highlighted 10 significant interactions with residues Asp1073.32, Tyr $108^{3.33}$, Ser111 $1^{3.36}$, Thr112 3.37 , Asn198 ${ }^{5.46}$, Phe1995.47, Trp428 ${ }^{6.48}$, Tyr431 $1^{6.51}$, Phe432 6.52 and Tyr458 ${ }^{7.43}$. The majority of these interactions are consistent with previously reported analysis [40]. Several hydrophobic interactions postulated from a previous report [40] (with lle115.40, Trp158.56, Thr $194^{5.43}$ and Phe435 ${ }^{6.55}$ ) were not detected as significant by FMO. The importance of hydrophobic interactions between Doxepin and $\mathrm{H}_{1}$, emphasized here by $\mathrm{FMO}$, are consistent with published observations that the tricyclic moiety of Doxepin adopts a deep binding position occupying a highly conserved hydrophobic pocket [34].

3.1. PIEDA calculations across all 18 GPCR-ligand systems showed [23] the utility of FMO and highlighted the importance of hydrophobic interactions, non-classical hydrogen bonds [14], interactions with the backbone atoms and water molecules for GPCRligand binding. According to this analysis, electrostatic and hydrophobic contributions to the ligand binding are approximately equal across all systems, on average [23].

3.2. We also observed [23] general trends in ligand binding for different proteins belonging to the same family. For example, the residues in positions $3.32,3.33,6.48,6.51,6.52$, 7.39 and 7.43 (located on TM3, TM6 and TM7) make considerable contributions to receptor-ligand binding and are quite conserved ( $>70 \%$, in the 18 tested cases). The residues in positions $3.33,6.48,6.51,6.52$ and 7.43 form interactions of a mainly hydrophobic nature while the residues in positions 3.32 and 7.39 form mainly electrostatic interactions. Published mutations in these positions frequently affect selectivity [41] and ligand binding affinity [42]. This observation is also consistent with a previous report [41] that residues in these positions highly frequently make contact with diverse ligands across nearly all class A GPCRs. Residues in the other positions are less frequently involved in interactions only with specific ligands [41]. These key positions in TM3, TM6 and TM7 form a consensus core of the ligand-binding pocket with any variation in the amino acids occupying the topologically equivalent positions 
contributing to ligand specificity in different GPCRs. This is in spite of the observation that the amino acids present in these positions are not conserved as been previously reported for 18 tested systems [23]. According to PIEDA, the predominant contribution in the majority of these conserved positions is, in fact, hydrophobic [23].

3.3. Overall, we have demonstrated how the FMO method can provide new insight into the nature of key chemical interactions required for receptor-ligand recognition. We anticipate that this approach can be used to provide insight into many protein-ligand interactions, and specifically to the design of new compounds.

\section{Comment:}

Some sections of this chapter are reprinted (adapted) with permission from the Journal of Chemical Information and Modeling 2016 Jan 25;56(1):159-72 [23]. Copyright (2019) American Chemical Society.

\section{ACKNOWLEDGMENT}

A.H. and A.T.N. would like to acknowledge the support of EU H2020 CompBioMed project (http://www.compbiomed.eu/, 675451) 


\section{References}

1. Hauser AS, Attwood MM, Rask-Andersen M, Schioth HB, Gloriam DE, (2017), Trends in GPCR drug discovery: new agents, targets and indications, Nat Rev Drug Discov. 16: 829-842.

2. Heifetz A, Schertler GF, Seifert R, Tate CG, Sexton PM, Gurevich VV, Fourmy D, Cherezov V, Marshall FH, Storer RI, Moraes I, Tikhonova IG, Tautermann CS, Hunt P, Ceska T, Hodgson S, Bodkin MJ, Singh S, Law RJ, Biggin PC, (2015), GPCR structure, function, drug discovery and crystallography: report from Academia-Industry International Conference (UK Royal Society) Chicheley Hall, 1-2 September 2014, Naunyn Schmiedebergs Arch Pharmacol.

3. Tautermann CS, (2014), GPCR structures in drug design, emerging opportunities with new structures, Bioorg Med Chem Lett. 24: 4073-4079.

4. Shonberg J, Kling RC, Gmeiner P, Lober S, (2015), GPCR crystal structures: Medicinal chemistry in the pocket, Bioorg Med Chem. 23: 3880-3906.

5. Jazayeri A, Dias JM, Marshall FH, (2015), From G Protein-coupled Receptor Structure Resolution to Rational Drug Design, J Biol Chem. 290: 19489-19495.

6. Bissantz C, Kuhn B, Stahl M, (2010), A medicinal chemist's guide to molecular interactions, J Med Chem. 53: 5061-5084.

7. Tong Y, Mei Y, Li YL, Ji CG, Zhang JZ, (2010), Electrostatic polarization makes a substantial contribution to the free energy of avidin-biotin binding, J Am Chem Soc. 132: 5137-5142.

8. Raha K, Peters MB, Wang B, Yu N, Wollacott AM, Westerhoff LM, Merz KM, Jr., (2007), The role of quantum mechanics in structure-based drug design, Drug Discov Today. 12: 725-731.

9. Beratan DN, Liu C, Migliore A, Polizzi NF, Skourtis SS, Zhang P, Zhang Y, (2015), Charge transfer in dynamical biosystems, or the treachery of (static) images, Acc Chem Res. 48: 474-481.

10. Ozawa T, Okazaki K, Kitaura K, (2011), CH/pi hydrogen bonds play a role in ligand recognition and equilibrium between active and inactive states of the beta2 adrenergic receptor: an ab initio fragment molecular orbital (FMO) study, Bioorg Med Chem. 19: 5231-5237.

11. Fedorov DG, Nagata T, Kitaura K, (2012), Exploring chemistry with the fragment molecular orbital method, Phys Chem Chem Phys. 14: 7562-7577.

12. Lu Y-X, Zou J-W, Wang Y-H, Yu Q-S, (2007), Substituent effects on noncovalent halogen/ $\pi$ interactions: Theoretical study, International Journal of Quantum Chemistry. 107: 1479-1486.

13. Gallivan JP, Dougherty DA, (1999), Cation-pi interactions in structural biology, Proc Natl Acad Sci U S A. 96: 9459-9464.

14. Johnston RC, Cheong PH, (2013), C-H...O non-classical hydrogen bonding in the stereomechanics of organic transformations: theory and recognition, Org Biomol Chem. 11: 50575064.

15. Yu N, Li X, Cui G, Hayik SA, Merz KM, 2nd, (2006), Critical assessment of quantum mechanics based energy restraints in protein crystal structure refinement, Protein Sci. 15: 2773-2784.

16. Fedorov DG, Kitaura K, (2007), Extending the power of quantum chemistry to large systems with the fragment molecular orbital method, J Phys Chem A. 111: 6904-6914.

17. Phipps MJ, Fox T, Tautermann CS, Skylaris CK, (2015), Energy decomposition analysis approaches and their evaluation on prototypical protein-drug interaction patterns, Chem Soc Rev. 44: 3177-3211.

18. Kitaura K, Ikeo E, Asada T, Nakano T, Uebayasi M, (1999), Fragment molecular orbital method: an approximate computational method for large molecules, Chemical Physics Letters. 313: 701-706. 19. Alexeev Y, Mazanetz MP, Ichihara O, Fedorov DG, (2012), GAMESS as a free quantummechanical platform for drug research, Curr Top Med Chem. 12: 2013-2033.

20. Fedorov DG, Kitaura K, (2007), Pair interaction energy decomposition analysis, J Comput Chem. 28: 222-237.

21. Barker JJ, Barker O, Courtney SM, Gardiner M, Hesterkamp T, Ichihara O, Mather O, Montalbetti CA, Muller A, Varasi M, Whittaker M, Yarnold CJ, (2010), Discovery of a novel Hsp90 inhibitor by fragment linking, ChemMedChem. 5: 1697-1700.

22. Sawada T, Fedorov DG, Kitaura K, (2010), Binding of influenza A virus hemagglutinin to the sialoside receptor is not controlled by the homotropic allosteric effect, J Phys Chem B. 114: 1570015705.

23. Heifetz A, Chudyk El, Gleave L, Aldeghi M, Cherezov V, Fedorov DG, Biggin PC, Bodkin MJ, (2016), The Fragment Molecular Orbital Method Reveals New Insight into the Chemical Nature of GPCR-Ligand Interactions, J Chem Inf Model. 56: 159-172.

24. Mazanetz MP, Ichihara O, Law RJ, Whittaker M, (2011), Prediction of cyclin-dependent kinase 2 inhibitor potency using the fragment molecular orbital method, J Cheminform. 3: 2. 
25. Fedorov DG, Kitaura K, (2012), Energy decomposition analysis in solution based on the fragment molecular orbital method, J Phys Chem A. 116: 704-719.

26. El Kerdawy A, Murray JS, Politzer P, Bleiziffer P, Hesselmann A, Gorling A, Clark T, (2013), Directional Noncovalent Interactions: Repulsion and Dispersion, J Chem Theory Comput. 9: 22642275.

27. Schmidt MW, Baldridge KK, Boatz JA, Elbert ST, Gordon MS, Jensen JH, Koseki S, Matsunaga N, Nguyen KA, Su S, Windus TL, Dupuis M, Montgomery Jr JA, (1993), General atomic and molecular electronic structure system, Journal of Computational Chemistry. 14: 1347-1363. 28. Tanaka S, Mochizuki Y, Komeiji Y, Okiyama Y, Fukuzawa K, (2014), Electron-correlated fragment-molecular-orbital calculations for biomolecular and nano systems, Phys Chem Chem Phys. 16: $10310-10344$.

29. Ishikawa T, Kuwata K, (2012), RI-MP2 Gradient Calculation of Large Molecules Using the Fragment Molecular Orbital Method, J Phys Chem Lett. 3: 375-379.

30. Nishimoto Y, Fedorov DG, (2017), Three-body expansion of the fragment molecular orbital method combined with density-functional tight-binding, J Comput Chem. 38: 406-418.

31. Fedorov DG, Kitaura K, (2004), Second order Moller-Plesset perturbation theory based upon the fragment molecular orbital method, J Chem Phys. 121: 2483-2490.

32. Zhang H, Unal H, Gati C, Han GW, Liu W, Zatsepin NA, James D, Wang D, Nelson G, Weierstall U, Sawaya MR, Xu Q, Messerschmidt M, Williams GJ, Boutet S, Yefanov OM, White TA, Wang C, Ishchenko A, Tirupula KC, Desnoyer R, Coe J, Conrad CE, Fromme P, Stevens RC, Katritch V, Karnik SS, Cherezov V, (2015), Structure of the Angiotensin Receptor Revealed by Serial Femtosecond Crystallography, Cell.

33. Tan Q, Zhu Y, Li J, Chen Z, Han GW, Kufareva I, Li T, Ma L, Fenalti G, Li J, Zhang W, Xie X, Yang H, Jiang H, Cherezov V, Liu H, Stevens RC, Zhao Q, Wu B, (2013), Structure of the CCR5 chemokine receptor-HIV entry inhibitor maraviroc complex, Science. 341: 1387-1390.

34. Shonberg J, Kling RC, Gmeiner P, Lober S, (2014), GPCR crystal structures: Medicinal chemistry in the pocket, Bioorg Med Chem.

35. Wang C, Jiang Y, Ma J, Wu H, Wacker D, Katritch V, Han GW, Liu W, Huang XP, Vardy E, McCorvy JD, Gao X, Zhou XE, Melcher K, Zhang C, Bai F, Yang H, Yang L, Jiang H, Roth BL, Cherezov V, Stevens RC, Xu HE, (2013), Structural basis for molecular recognition at serotonin receptors, Science. 340: 610-614.

36. Kruse AC, Hu J, Kobilka BK, Wess J, (2014), Muscarinic acetylcholine receptor X-ray structures: potential implications for drug development, Curr Opin Pharmacol. 16: 24-30.

37. Dougherty DA, (2013), The cation-pi interaction, Acc Chem Res. 46: 885-893.

38. Kruse AC, Hu J, Pan AC, Arlow DH, Rosenbaum DM, Rosemond E, Green HF, Liu T, Chae PS, Dror RO, Shaw DE, Weis WI, Wess J, Kobilka BK, (2012), Structure and dynamics of the M3 muscarinic acetylcholine receptor, Nature. 482: 552-556.

39. Tautermann CS, Kiechle T, Seeliger D, Diehl S, Wex E, Banholzer R, Gantner F, Pieper MP, Casarosa P, (2013), Molecular basis for the long duration of action and kinetic selectivity of tiotropium for the muscarinic M3 receptor, J Med Chem. 56: 8746-8756.

40. Shimamura T, Shiroishi M, Weyand S, Tsujimoto H, Winter G, Katritch V, Abagyan R, Cherezov V, Liu W, Han GW, Kobayashi T, Stevens RC, Iwata S, (2011), Structure of the human histamine H1 receptor complex with doxepin, Nature. 475: 65-70.

41. Venkatakrishnan AJ, Deupi X, Lebon G, Tate CG, Schertler GF, Babu MM, (2013), Molecular signatures of G-protein-coupled receptors, Nature. 494: 185-194.

42. Dore AS, Robertson N, Errey JC, Ng I, Hollenstein K, Tehan B, Hurrell E, Bennett K, Congreve M, Magnani F, Tate CG, Weir M, Marshall FH, (2011), Structure of the adenosine $A(2 A)$ receptor in complex with ZM241385 and the xanthines XAC and caffeine, Structure. 19: 1283-1293. 